\title{
PAPER
}

\section{Effect of brain tumour laterality on patients' perceived quality of life}

\author{
J Salo, A Niemelä, M Joukamaa, J Koivukangas
}

J Neurol Neurosurg Psychiatry 2002;72:373-377

See end of article for authors' affiliations

.....................

Correspondence to: Dr A Niemelä, Department of Neurosurgery, Oulu University Hospital, PL 22, 90220 Oulu, Finland; asko.niemela@ppsh.fi

Received 6 April 2001

In revised form

2 October 2001

Accepted

11 October 2001

....................

\begin{abstract}
Objectives: There is little reliable quantitative information on preoperative quality of life of patients with brain tumours. The aim of this study was to clarify the effect of the volume, location, and histological grade of brain tumours on the preoperative quality of life of patients.

Methods: The study population consisted of 101 successive patients with brain tumour at Oulu Clinic for Neurosurgery studied with CT or MRI for preoperative determination of tumour location and size. The Nottingham health profile (NHP) and Sintonen's 15D scale were used at that time to measure quality of life.

Results: Tumour size did not correlate linearly with impairment of quality of life. Large tumours (>25 $\mathrm{ml}$ ) were associated with poorer quality of life than small tumours ( $\leqslant 25 \mathrm{ml})$. The patients with a tumour located on the right side or in the anterior region reported a poorer quality of life than those with a tumour on the left side or posteriorly. Quality of life assessments made by doctors using the Karnofsky performance scale showed no differences between the two hemispheres. Patients with the most malignant gliomas (grades III-IV) displayed the poorest quality of life.

Conclusions: Large tumours apparently damage several parts of the brain and/or raise intracranial pressure to a level that exceeds the brain's compensatory capacity. Contrary to earlier understanding, tumours in the right hemisphere seemed to be related to poorer quality of life. This effect was especially clear in the patients' subjective evaluation of their quality of life. As the location of the brain tumour thus affects perceived quality of life, any measurements of the quality of life of patients with brain tumours should take into account the location and laterality of the tumour.
\end{abstract}

$\mathrm{T}$ he effects of a brain tumour on the patients' subjective quality of life are mediated by many different mechanisms. Depending on the histological grade, there are differences in the mode of tumour spreading (infiltration/ displacement of cerebral tissue/metastasis), the rate of growth, and the extent of oedema surrounding the tumour. Tumour volume also causes a rise in intracranial pressure ${ }^{1}$ whereas tumour location affects the quality of specific symptoms.

The effects of the location, volume, and histological grade of the tumour on the patients' preoperative quality of life and its different dimensions are not well known, nor is it known which of these dimensions is most relevant in practice. One of the obvious problems is that very few studies have been done with validated instruments measuring quality of life $\mathrm{e}^{2-4}$ including the different subdomains of health related quality of life (physical, psychological, social, and functional). The older studies have especially concentrated exclusively on measuring the physical component, mostly with the Karnofsky performance scale (KPS). ${ }^{5}$ Many of the existing studies have also centred on the postoperative situation, ${ }^{23}$ when in addition to the stress caused by the serious illness, surgery, and other treatments can in themselves affect quality of life.

The purpose of this study was to find answers to the following questions: (1) How are the size, location, and histological grade of the brain tumour related to the patient's subjective health related quality of life preoperatively? (2) Which of these factors is most significant in terms of the patient's quality of life? (3) How does the brain tumour affect the patient's perception of his or her quality of life?

From previous studies we know that as far as speech, mood, ${ }^{6-9}$ use of the right hand, and postoperative quality of life $^{23}$ are concerned, damage or tumour in the left hemisphere affects the patient more than damage in the right hemisphere. Therefore our main hypothesis was that the patients who have a tumour in the left hemisphere have a poorer quality of life than those with a tumour in the right hemisphere.

\section{PATIENTS AND METHODS}

The study population consisted of 101 successive patients with brain tumours treated surgically at the Oulu Clinic for Neurosurgery, Oulu University Hospital, during February 1990 to March 1992. Patients aged under 16 years and those with metastases had been excluded from the series as had eight patients who did not give informed consent (one patient with acoustic neurinoma, two with meningeoma, five with glioma). Brain CT or MRI were not available for four patients, leaving 97 patients for further study. The primary mode of treatment in all these cases was surgery.

Epidemiologically, the series is a representative and unselected cohort, because the Oulu Clinic for Neurosurgery admits all the surgically treatable patients with brain tumour in its catchment area. Moreover, all adult patients with primary brain tumours successively admitted and surgically treated during the study period were included.

The demographic patient data and the distribution of tumours based on location and histological grade are shown in table 1. Histological grading was done according to the World Health Organisation (WHO) classification. ${ }^{10}$ The tumours were divided into six classes: glioma grade I-II, glioma grade III-IV, meningioma, pituitary adenoma, acoustic neurinoma, and other tumours (table 1).

The patients underwent preoperative CT or MRI. Brain CT was the most often used diagnostic imaging method at that

Abbreviations: NHP, Nottingham Health profile; KPS, Karnofsky performance scale 
Table 1 Patients with brain tumour and the location, size, and histological diagnosis of the tumours

\begin{tabular}{ll}
\hline Sex: & \\
Male & $39(39)$ \\
Female & $62(61)$ \\
Mean age (y) & 49 \\
Range (y) & $20-82$ \\
Location of tumour (n=97): & \\
Left & $45(46)$ \\
Anterior & $23(51)$ \\
Posterior & $16(36)$ \\
Right & $34(35)$ \\
Anterior & $15(44)$ \\
Posterior & $10(29)$ \\
Bilateral & $14(14)$ \\
Anterior & $11(79)$ \\
Posterior & $1(7)$ \\
Undefined location & $4(4)$ \\
Tumour volume: & \\
Mean $45 \mathrm{ml}$ & \\
Under $25 \mathrm{ml}$ & $50 \%$ \\
Over $55 \mathrm{ml}$ & $25 \%$ \\
Diagnosis: & $19(19)$ \\
Grade I-II glioma (V=42 ml) & $22(22)$ \\
Grade III-IV glioma (V=37 ml) & $33(33)$ \\
Meningioma (V=23 ml) & $8(8)$ \\
Pituitary adenoma (V=8 ml) & $13(13)$ \\
Acoustic neurinoma (V=4 ml) & $6(6)$ \\
Other* (V=18 ml) & \\
\hline
\end{tabular}

Values in parentheses are \%; $V=$ mean volume of tumours *Hemangiopericytoma (two); malignant lymphoma; * Hemangiopericytoma (two); maligna

time. Magnetic resonance imaging was carried out on 16 $(16 \%)$ patients. Matsui's anatomical classification of the brain was used to define the location of various brain structures. ${ }^{11}$ The tumours were divided into ones located in the left or right hemisphere and anteriorly or posteriorly, if they reached the supratentorial space. The distance of the tumour from the apex of the frontal lobe was determined by calculating from each CT or MRI slice the ratio of the distance between the anterior part of the tumour and the apex of the frontal lobe to the anterior-posterior diameter of the whole brain. The mean of these percentages was used to describe the distance from the apex of the frontal lobe to the tumour images.

Moreover, each hemisphere was divided, in accordance with the model of Levine and Grek, ${ }^{12}$ into 12 areas: central gyri, superior frontal gyrus, frontotemporal region, superior parietal lobule, basal temporo-occipital region, occipital convexity, lenticular nucleus, caudate nucleus, thalamus, posterior limb of internal capsule, anterior limb of internal capsule, and temporoparietal region. The volume of each brain tumour was determined manually from the CT or MRI.

The patients' quality of life was measured preoperatively with Sintonen's 15D scale ${ }^{13} 14$ and the Nottingham health profile (NHP). ${ }^{15}$ Both of these are general quality of life instruments, not specifically designed or standardised for patients with brain tumours. The 15D scale is a self report questionnaire that measures quality of life on 15 different dimensions (breathing, mental functions, speech, vision, mobility, working capacity, subjective health, hearing, eating, continence, sleep, anxiety, pain and ache, social participation, and depression). The dimensions are ranked relative to each other, and by summing up their scores, it is possible to obtain a value of $0-1$, which represents the patient's overall quality of life. A completely healthy person in good condition thus scores 1 on the $15 \mathrm{D}$ scale. ${ }^{13}$

The NHP is also a self report questionnaire, which consists of two parts. The first part includes 38 weighted yes/no questions, which yield quality of life scores of $0-100$ on six dimensions (energy, pain, sleep, social isolation, emotional reactions, and mobility). The second part consists of seven statements pertaining to problems caused by health status, to which the patient replies yes or no. The NHP hence does not yield a single score but rather a profile of the patient's quality of life. The NHP version standardised in Finland was used. ${ }^{16}$

The patient's condition was also evaluated on the KPS scale, which is an ordinal scale of functional status. The KPS categorises the patients from 0 (dead) to 100 (healthy) at 10 unit intervals and is rated by the physician. The scale emphasises the presence of symptoms, ability to work, physical activity, and self care. ${ }^{5}$

Statistical analysis was done with the SPSS software, using as independent variables the location of the brain tumour (right or left hemisphere, anterior or posterior region), its distance from the apex of the frontal lobe, tumour volume, size class, and histological grade. The tumours were divided into two size classes ( $\leqslant 25 \mathrm{ml} ;>25 \mathrm{ml}$ ). The significance of size was analysed further by dividing the larger tumours into two subclasses ( size $>25 \mathrm{ml}$ but $<55 \mathrm{ml}$ or $\geqslant 55 \mathrm{ml}$ ). Because, apart from the KPS, the distributions were skewed, the results are presented as medians, and non-parametric tests (MannWhitney $U$ and Kruskal-Wallis $H$ ) were used in the statistical analysis. The statistical significance of differences in KPS was determined with the $t$ test. The correlations were assessed with Pearson's correlation coefficient.

\section{RESULTS}

Tumour volume and quality of life did not correlate linearly. Small $(\leqslant 25 \mathrm{ml})$ tumours were associated with better quality of life in measurements with both 15D and NHP (table 2) than large (>25 ml) ones. However, no differences in quality of life were seen between the groups with tumour volumes of less than $25 \mathrm{ml}$ and those with volumes of 25 to $55 \mathrm{ml}$, which means that the statistical difference was due to the poor quality of life in the subset of the patients with very large tumours.

Large tumours $(>25 \mathrm{ml}$ ) located in the left hemisphere did not impair quality of life any more than did the small tumours ( $\leqslant 25 \mathrm{ml}$ ), whereas in the right hemisphere the large tumours were associated with a statistically significantly poorer quality of life on all the other dimensions of NHP except sleep and emotional reactions.

The patients with a tumour in the right hemisphere had poorer quality of life on both 15D and NHP than did those with a tumour on the left side (table 2). On the 15D scale, the means of all dimensions except speech were poorer for the patients with a tumour in the right hemisphere. When this difference was analysed by dividing the brain into smaller areas, ${ }^{12}$ it turned out that in nine of the 12 areas, a tumour located in the right hemisphere impaired quality of life more than in the corresponding location in the left hemisphere. The only areas where the effect of the tumour on the 15D index was not bigger in the right hemisphere were the occipital convexity, the basal temporo-occipital region, and the superior parietal region. In all the lateral diagnostic categories, the mean of the 15D indexes was poorer for those patients with a tumour in the right hemisphere. Average tumour size did not differ between the hemispheres, nor did the patients' age or sex.

The location of the tumour on the anterior-posterior axis also affected quality of life. Anterior tumours had a lower than average 15D index (table 2). The NHP also yielded a poorer quality of life for anterior tumours, and the difference was significant on the NHP dimensions of sleep and social relations. The patients with an anterior tumour in the right hemisphere had the poorest quality of life, whereas those with an anterior tumour in the left hemisphere had the best quality of life. The distance between the brain tumour and the apex of the frontal lobe, on the other hand, did not correlate linearly $(r<0.3)$ with either the patient's quality of life or the KPS. The KPS value was about the same, regardless of which hemisphere the tumour was located in (80 on the left $v 76$ on the right, $\mathrm{p}=0.28$ ). 
Table 2 Differences in quality of life as measured by Sintonen's 15D and Nottingham health profile (NHP) (median values) in the different patient groups: tumour size ( $\leqslant 25 \mathrm{ml} v>25 \mathrm{ml}$ ), tumour location (left $v$ right hemisphere and anterior $v$ posterior)

\begin{tabular}{|c|c|c|c|c|c|c|c|c|c|}
\hline & \multicolumn{3}{|c|}{ Tumour size } & \multicolumn{3}{|c|}{ Affected hemisphere } & \multicolumn{3}{|c|}{ Location on the anterior-posterior axis } \\
\hline & $\begin{array}{l}\leqslant 25 \mathrm{ml} \\
(\mathrm{n}=47)\end{array}$ & $\begin{array}{l}>25 \mathrm{ml} \\
(\mathrm{n}=45)\end{array}$ & $\mathrm{p}$ Value & Left $(n=43)$ & $\begin{array}{l}\text { Right } \\
(n=33)\end{array}$ & $\mathrm{p}$ Value & $\begin{array}{l}\text { Anterior } \\
(n=47)\end{array}$ & $\begin{array}{l}\text { Posterior } \\
(n=32)\end{array}$ & $\mathrm{p}$ Value \\
\hline Sintonen's 15D & 0.90 & $0.84(\mathrm{~N}=48)$ & 0.062 & 0.91 & $0.82(n=34)$ & $0.007 * *$ & $0.86(n=48)$ & 0.91 & 0.659 \\
\hline NHP: pain & 0.0 & 9.1 & $0.035^{*}$ & 0.0 & 10.3 & $0.003 * *$ & 0.0 & 0.0 & 0.763 \\
\hline NHP: mobility & 0.0 & 9.0 & $0.008 * *$ & 0.0 & 0.0 & 0.116 & 0.0 & 0.0 & 0.722 \\
\hline NHP: social isolation & 0.0 & 16.4 & $0.013^{*}$ & 0.0 & 16.4 & $0.014^{*}$ & 0.0 & 0.0 & $0.043^{*}$ \\
\hline NHP: energy & 0.0 & 24.8 & $0.023^{*}$ & 0.0 & 24.8 & 0.112 & 0.0 & 0.0 & 0.346 \\
\hline NHP: emotion & 0.0 & 6.2 & 0.189 & 0.0 & 8.0 & $0.048 *$ & 0.0 & 0.0 & 0.730 \\
\hline NHP: sleep & 18.1 & 27.0 & 0.206 & 6.9 & 28.9 & 0.086 & 28.9 & 6.9 & $0.003 *$ * \\
\hline
\end{tabular}

The patients with glioma grades III - IV had the poorest quality of life on both 15D and all dimensions of NHP except sleep, whereas the patients with acoustic neurinoma had the best average quality of life (table 3). Using Kruskal-Wallis $H$ analysis, the differences were significant only on the NHP dimensions of pain and social functions. When the tumour was located in the left hemisphere, none of the NHP dimensions were significantly related to the diagnosis, whereas the patients with a tumour in the right hemisphere had significant differences on the dimensions of social functions $(p=0.021)$ and energy $(p=0.009)$. The patients with glioma grades III-IV were in the poorest condition. The differences between the 15D medians of the histological groups among the patients with a tumour on the left side were much smaller than the corresponding differences among those with a tumour on the right side. However, none of these differences were significant.

\section{DISCUSSION}

As far as we know, there are no previous quantitative studies on the effects of brain tumours on the patients' preoperative quality of life. According to the main findings of this study, the patients with a tumour on the right side had poorer quality of life than those with a tumour on the left side. The patients with an anterior tumour similarly had poorer quality of life than those with a posterior tumour. Parallel differences were seen in all tumour categories. There was no direct correlation between tumour volume and the 15D or NHP dimensions, but very large tumours involved clearly impaired quality of life.

The postoperative study by Giovagnoli et al showed tumour laterality to have an effect: the patients with an anterior tumour in the right hemisphere had a better quality of life. ${ }^{2}$ Furthermore, trauma to the motor cortex of the left hemisphere caused more inconvenience to right handed patients than corresponding trauma in the right hemisphere. Studies on patients with cerebral haemorrhage and trauma have shown that damage to the anterior part of the left hemisphere affects mood more than a corresponding injury to the right hemisphere..$^{6-9}$ Trauma on the left side affects linguistic capabilities more than trauma on the right, whereas trauma on the right side delays social recovery more than trauma on the left. ${ }^{17}$ We therefore hypothesised that patients with a tumour in the left hemisphere would have poorer subjective quality of life than those with a tumour on the right side. Other hypotheses were that large tumours would impair the patients' quality of life more than small tumours and that the patients with malignant tumours would have poorer quality of life than the other patients.

Brain CT was used as the primary investigation to obtain maximally consistent data, as CT was the most commonly used diagnostic imaging method in this series. Tumour size and location were defined manually from the scans. If MRI had been available for all patients, it would have been possible to determine tumour size and location more precisely, but this would not have affected the important issue of laterality. It would also have been possible to make more precise determinations if the images had been stored digitally. Despite these shortcomings, the results can be considered reliable, for the accuracy of CT and the method of measurement are quite adequate in view of the main findings. The method used here to determine the location of the brain tumour and its distance from the frontal lobe is the one most commonly used in studies of the connections between cerebral haemorrhages and traumas on the one hand and mood disorders and recovery from them on the other. ${ }^{618-20}$ Our aim was thus to obtain results comparable with the previous findings.

The effect of tumour location in this study was opposite to that reported by Giovagnioli et al, ${ }^{2}$ which may be, at least partly, explained by the different instrument used and the different timing of the examinations (their's were made postoperatively). The effect of the affected hemisphere on the 15D and NHP scores can be explained by one of the following postulations: (a) injury to the right hemisphere truly impairs

Table 3 Quality of life measured by Sintonen's 15D and Nottingham health profile (NHP) by histological diagnosis (median values)

\begin{tabular}{|c|c|c|c|c|c|c|c|}
\hline & \multicolumn{2}{|l|}{ Glioma } & \multirow{2}{*}{$\begin{array}{l}\text { Acoustic } \\
\text { neurinoma } \\
(n=13)\end{array}$} & \multirow[b]{2}{*}{$\begin{array}{l}\text { Meningioma } \\
(n=31)\end{array}$} & \multirow[b]{2}{*}{$\begin{array}{l}\text { Pituitary } \\
\text { adenoma }\end{array}$} & \multirow[b]{2}{*}{ Other } & \multirow[b]{2}{*}{$\mathrm{p}$ Value } \\
\hline & $\begin{array}{l}\text { Grades I-II } \\
(n=19)\end{array}$ & $\begin{array}{l}\text { Grades III-IV } \\
(n=20)\end{array}$ & & & & & \\
\hline Sintonen's 15D & 0.92 & 0.84 & 0.89 & $0.86(\mathrm{~N}=32)$ & 0.88 & 0.87 & 0.613 \\
\hline NHP: pain & 0.0 & 22.3 & 0.0 & 8.8 & 0.0 & 9.1 & $0.035^{*}$ \\
\hline NHP: mobility & 0.0 & 9.0 & 0.0 & 0.0 & 0.0 & 9.0 & 0.148 \\
\hline NHP: social isol. & 0.0 & 16.4 & 0.0 & 0.0 & 0.0 & 17.1 & $0.025^{*}$ \\
\hline NHP: energy & 0.0 & 29.6 & 0.0 & 0.0 & 0.0 & 24.8 & 0.250 \\
\hline NHP: emotion & 0.0 & 4.0 & 8.0 & 6.2 & 3.1 & 0.0 & 0.566 \\
\hline NHP: sleep & 6.9 & 28.9 & 0.0 & 27.0 & 32.4 & 35.8 & 0.244 \\
\hline
\end{tabular}


quality of life more than corresponding injury on the left side, (b) people with a brain tumour in the right hemisphere perceive their quality of life as poorer than those with a tumour on the left, (c) patients with a brain tumour on the left side perceive their quality of life to be better than an outsider's assessment, or $(d)$ an interfering variable confounds the findings.

It is probable that item (c) is right. As many important functions, such as speech and the right hand motor area, are located on the left side, it is difficult to assume that patients with a left sided brain tumour would truly have better quality of life than even a sample of general patients visiting a health centre (15D index 0.88). ${ }^{21}$ Moreover, the patients with a tumour in the left hemisphere reported more or less the same quality of life regardless of tumour size, whereas the patients with a tumour on the right side had clearly poorer quality of life when the tumour was large. The correlation with the histological diagnoses was parallel: in the case of left hemisphere tumours, quality of life showed weak, if any, correlation with the malignancy of the tumour, whereas increasing malignancy of tumours on the right side resulted in clearly poorer quality of life. It is also noteworthy that tumours in the left hemisphere were associated with better scores on all the 15D and NHP dimensions except speech on 15D, which suggests that these tumours result in profound cerebral disorder instead of merely affecting certain functions located in particular areas of the brain. The degree of invasion/volume of the tumour could be assumed to be a confounding factor, as injury to the left hemisphere causes earlier and more distinct damage, but there was no significant difference in tumour volume between the hemispheres, nor was there difference in the patients' sex or average age.

Why, then, do patients with a brain tumour on the left side evaluate their quality of life as better than an outsider's estimate, and how big is this difference? The reason may be a cognitive inability to evaluate one's condition and to reply to questions coherently. According to some researchers, the human ego and consciousness are mostly located in the left hemisphere, ${ }^{22}{ }^{23}$ which implies that injury to this hemisphere could impair the patients' ability to evaluate their quality of life and functional capacity. Another potential explanation could be that a pathologically positive mood gives rise to an unrealistic understatement of problems and complaints. This explanation is not unambiguous, however, as previous research has disclosed a correlation between depression and injury to the anterior part of the left hemisphere, ${ }^{6-9} 1819$ whereas our findings suggest a correlation between this tumour location and a maximally good quality of life. Furthermore, the right hemisphere has been associated with negative feelings, ${ }^{22}$ and injury to the right hemisphere has been found to cause apathy. All in all, the present finding is unexpected and suggests a need for further research.

The connection between very large tumours and impaired quality of life may be explained by the injurious effects of large tumours on several parts of the brain. The brain is able to compensate for the increase of mass due to small tumours, whereas large tumours raise intracranial pressure enough to exceed the compensatory capacity of the brain. The effects of the location, volume and histological grade of brain tumours hence differed from each other. Sleep disorders, for example, were clearly connected to anterior tumours, whereas tumour volume and histological grade had practically no correlation with sleep disorders, and which hemisphere was affected showed no statistical correlation. Also, location on the anterior-posterior axis and histological grade seemed to have more specific effects in the right hemisphere than did large volume or location, which generally affected all dimensions of quality of life. The effect of anterior tumours on sleep and social functions may be explained by the restlessness and personality change caused by them, whereas the effect of histological grade on pain could be explained by the rapid growth of the most malignant tumours and their infiltration into pain sensitive tissues. As was already pointed out above, large tumours and those located in the left hemisphere cause generalised brain malfunction.

We can hence answer our first question by saying that very large tumours impair all dimensions of quality of life, and that location in the anterior region or in the right hemisphere and malignant grade are also related to poor quality of life. Specific tumour locations on the anterior-posterior axis give rise to different dysfunctions. The most important qualities of malignant tumours from the viewpoint of subjective quality of life seem to be their mode and rate of growth. Our second and third questions can be answered by saying that the most important single factor that affects the patient's quality of life seems to be the hemisphere afflicted by the tumour. Thus, a tumour in the left hemisphere often makes it difficult to measure reliably the patient's preoperative quality of life, as it may distort the patient's subjective assessment of him/herself.

The present results are, in many respects, unexpected and contradictory to previous findings. We intend to further elucidate the mechanisms whereby brain tumours impair the patient's quality of life by comparing the postoperative recovery of patients to the results obtained by measuring health status with various instruments (neurological, mental, neuropsychological). This will help us to find out if the situation is different postoperatively, as suggested by our preliminary results. At any rate, we are faced by an interesting problem, which should be solved to gain better insight into both brain function and the development of quality of life measurements.

\section{Authors' affiliations}

J Salo, A Niemelä, M Joukamaa, J Koivukangas, Department of Neurosurgery, Oulu University Hospital, PL 22, 90220 Oulu, Finland

\section{REFERENCES}

1 Boucher Y, Salehi H, Witwer B, et al. Interstitial fluid pressure in intracranial tumours in patients and in rodents. $\mathrm{Br} J$ Cancer 1997;75:829-36

2 Giovagnioli AR, Tamburini M, Boiardi A. Quality of life in brain tumour patients. J Neurooncol 1996;30:71-80.

3 Weitzner MA, Meyers CA, Byrne K. Psychosocial functioning and quality of life in patients with primary brain tumours. J Neurosurg 1996:84:29-34.

4 Koivukangas $\mathbf{P}$, Koivukangas J. Role of quality of life in therapeutic strategies in brain tumors. Health Policy 1988;10:241-57.

5 Karnofsky DA, Abelmann WH, Craver LF, et al. The use of nitrogen mustards in palliative treatment of carcinoma. Cancer 1948;1:634-56. 6 Fedoroff JP, Starkstein SE, Forrester AW, et al. Depression in patients with acute traumatic brain injury. Am J Psychiatry 1992;149:918-23.

7 Jorge RE, Robinson RG, Starkstein SE, et al. Depression and anxiety following traumatic brain injury. J Neuropsychiatry Clin Neurosci 1993b;5:369-74.

8 Lipsey JR, Robinson RG, Pearlson GD, et al. Mood change following bilateral hemisphere brain injury. BrJ Psychiatry 1983;143:266-73.

9 Parikh RM, Lipsey JR, Robinson RG, et al. Two year longitudinal study of post-stroke mood disorders: dynamic changes in correlates of depression at one and two years. Stroke 1987; 18:579-84.

10 Kleihues P, Burger PC, Scheithaver BW. Histological typing of tumours of the central nervous system. WHO Classifications. 2nd ed. Berlin: Springer Verlag, 1993.

11 Matsui T, Kawamoto K, Iwata M, et al. Anatomical and pathological study of the brain by CT scanner. 1: Anatomical study of normal brain. Computerized Tomography 1977;1:3-43.

12 Levine DN, Grek A. The anatomic basis of delusions after right cerebral infarction. Neurology 1984;34:577-82.

13 Sintonen H, Pekurinen M. A fifteen dimensional measure of health-related quality of life (15D) and its applications. In: Walker S, Rosser R, (eds). Quality of life assessment: key issues in the 1990s. Dordrecht: Kluwer Academic Publisher, 1993:185-96.

14 Sintonen H. An approach to measuring and valuing health states. Soc Sci Med 1981;15:55-65.

15 Hunt SM, McKenna SP, et al. The Nottingham health profile: a subjective health status and medical consultations. Soc Sci Med $1981 ; 15: 221-9$

16 Koivukangas P, Ohinmaa A, Koivukangas J. Nottingham Health Profilen suomalainen version. (The Finnish version of the Nottingham health profile, with English summary). Helsinki: STAKES, 1995. (Report 187.)

17 Jorge R, Robinson R, et al. Influence of depression on 1-year outcome in patients with traumatic brain injury. J Neurosurg 1994;81:726-33. 
18 Jorge RE, Robinson RG, Arndt SV, et al. Depression following traumatic brain injury: 1 year longitudinal study. J Affect Disord 1993a;27:233-43

19 Robinson RG, Boston JD, et al. Comparison of mania and depression after brain injury: causal factors. Am J Psychiatry 1988:145:172-8.

20 Starkstein SE, Robinson RG, Honig MA, et al. Mood changes after right-hemisphere lesions. Br J Psychiatry 1989;155:79-85.
21 Sintonen H, Pekurinen M. (A Generic 15-Dimensional Measure of Health-Related Quality of Life (15D)). Journal of Social Medicine 1989;26:85-96. (In Finnish.)

22 Dimond S. Neuropsychology. A textbook of systems and psychological functions of the human brain. London: Butterworths, 1980.

23 Popper KR, Eccles JC: The self and its brain. Berlin: Springer International, 1977

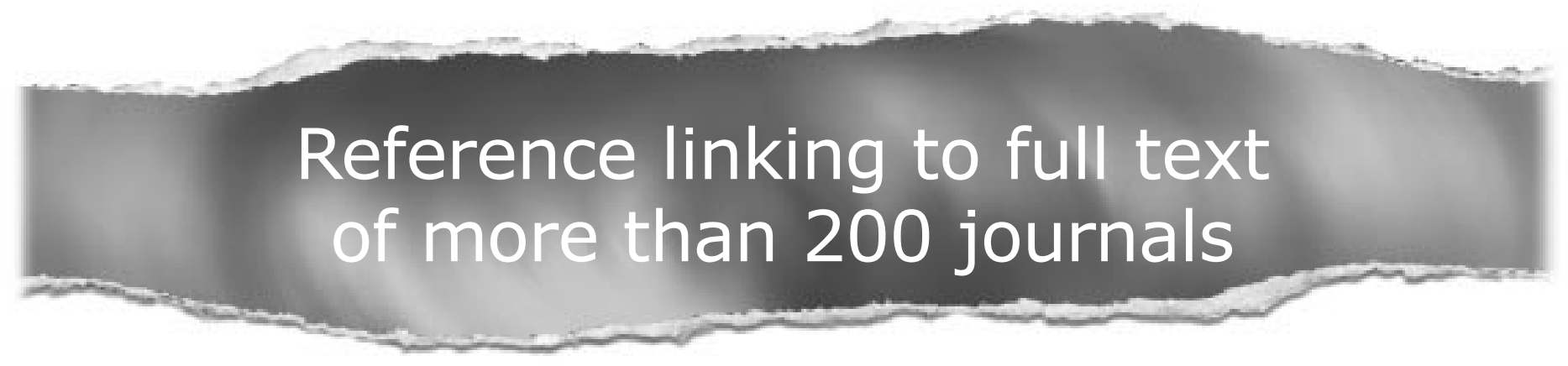

\section{Toll free links}

You can access the FULL TEXT of articles cited in the Journal of Neurology, Neurosurgery, and Psychiatry online if the citation is to one of the more than 200 journals hosted by HighWire (http://highwire.stanford.edu) without a subscription to that journal. There are also direct links from references to the Medline abstract for other titles.

www.jnnp.com 